E3S Web of Conferences 1, 21004 (2013)

DOI: $10.1051 / \mathrm{e} 3$ sconf/20130121004

(c) Owned by the authors, published by EDP Sciences, 2013

\title{
Search for cellular stress biomarkers in lymphocytes from individuals exposed to arsenic: a pilot study
}

\author{
A. I. Scovassi ${ }^{1}$, S. Grecchi ${ }^{1}$, L. Cori ${ }^{2}$ and F. Bianchi ${ }^{2}$ \\ ${ }^{1}$ Institute of Molecular Genetics, National Research Council, Pavia, Italy, scovassi@igm.cnr.it; \\ sabrina.grecchi@gmail.com \\ ${ }^{2}$ Unit of Environmental Epidemiology, Institute of Clinical Physiology, National Research Council, Pisa, Italy, \\ liliana.cori@ifc.cnr.it; fabrizio.bianchi@ifc.cnr.it
}

\begin{abstract}
The molecular mechanisms of arsenic toxicity are not fully elucidated. The Italian project SEpiAs aims at developing and validating the assay to detect stress biomarkers in individuals living in areas characterized by either natural or industrial arsenic contamination.
\end{abstract}

Key words: Apoptosis, arsenic, biomonitoring, cellular stress, DNA damage

\section{Introduction}

Inorganic arsenic and arsenic compounds have been classified in Group 1 "carcinogenic to humans" by IARC (2004).

Both short- and long-term exposure to arsenic can cause several health problems, including noxious effects on cardiovascular and neurological systems (Salnikov, 2008; Sturchio et al., 2009).

Arsenic and many of its compounds are potent poisons. Their mechanisms of action on cells include heavy inhibitory effects on ATP production, oxidative phosphorylation, and, in turn, mitochondrial respiration, because of the production of reactive oxygen species and oxidative stress. Finally, arsenic was found to induce cell death (Marchiset-Ferlay et al., 2012).

The careful examination of the molecular impact of arsenic implies a project of Human biomonitoring, that is the discipline devoted to the identification of biomarkers. The goal of this approach is to measure environmental exposure, to monitor biological effects and causal relationships to pathological conditions, and to possibly define the genetic susceptibility of the general population (Leoni and Scovassi, 2010).

To investigate the molecular impact of arsenic on cell metabolism, we started human biomonitoring in Italy on behalf of the Italian Ministry of Health (project SEpiAs CCM 2010).

The study is performed on adult population samples from individuals recruited in two areas with high levels of arsenic due to natural geological contamination, and two areas with the presence of arsenic of industrial origin.

We considered different biomarkers of arsenic exposure, i.e. the occurrence of DNA damage, cellular stress conditions and cell death (mainly apoptosis).

The biological material used in the study is represented by lymphocytes isolated from peripheral blood of individuals resident in "hot" geographic areas.

The evaluation of DNA damage is based on the analysis of the phosphorylation of histone H2AX on Serine139, which is a typical marker of DNA breaks (Kuo and Yang, 2008). The procedure consists in immunofluorescence experiments conducted with a specific antibody against the phosphorylated form of H2AX.

Cellular stress conditions can be monitored by measuring the synthesis of the polymer poly(ADP-ribose), which represents a valuable marker of endogenous-exogenous stress (Bürkle, 2001). The use of a monoclonal antibody against the polymer renders the immunofluorescence assay extremely specific to detect cellular stress conditions.

The possible induction of cell death is addressed by searching for a series of morphological and biochemical parameters, according to routinely used protocols (Scovassi et al., 2008).

The statistical analysis of the collected data will allow the evidence of the correlation (if any) of damage values and arsenic exposure. The results of this survey could help in understanding if arsenic toxicity is correlated with a net DNA damage and/or detectable cellular stress conditions. 


\section{Acknowledgements}

The authors thank the financial support from the Italian Ministry of Health (Project SEpiAs CCM 2010).

\section{References}

Bürkle A. Poly(ADP-ribosyl)ation, a DNA damage-driven protein modification and regulator of genomic instability. Cancer Lett 2001; 163:1-5.

IARC Some Drinking-water Disinfectants and Contaminants, including Arsenic. Monographs on the Evaluation of Carcinogenic Risks to Humans 2004; 84.

Kuo LJ, Yang LX. Gamma-H2AX - a novel biomarker for DNA double-strand breaks. In Vivo 2008; 22:305-309.

Leoni E, Scovassi AI. Human Biomonitoring. In CNR Environment and Health Inter-departmental Project: present knowledge and prospects for future research.
In: Bianchi F, Cavarretta G, Cori L, editors. CNR, Rome; 2010. p. 111-128.

Marchiset-Ferlay N, Savanovitch C, Sauvant-Rochat MP. What is the best biomarker to assess arsenic exposure via drinking water? Environ Int 2012; 39:150-171.

Salnikow K. Genetic and Epigenetic Mechanisms in Metal Carcinogenesis and Cocarcinogenesis: Nickel, Arsenic, and Chromium. Chem Res Toxicol 2008; 2:28-44.

Scovassi AI, Bottone MG, Biggiogera M, Pellicciari C. Dynamic relocation of nuclear proteins during the execution phase of apoptosis. Biochem Pharmacol 2008; 76:1440-1450.

Sturchio E, Minoia C, Zanellato Masotti A, Leoni E, Sottani C, Biamonti G, Ronchi A, Casorri L, Signorini S, Imbriani M. Endocrine disruptors Monograph 3. Arsenic. G Ital Med Lav Ergon. 2009; $31: 5-32$ 